\title{
Irradiation tests and expected performance of readout electronics of the ATLAS hadronic endcap calorimeter for the HL-LHC
}

\author{
Alexander Cheplakov, on behalf of the ATLAS Liquid Argon Calorimeter Group \\ Laboratory of High Energy Physics, JINR \\ 141980 Dubna, Russia \\ E-mail: Alexander.Cheplakovecern.ch
}

\begin{abstract}
The readout electronics of the ATLAS Hadronic Endcap Calorimeter (HEC) will have to withstand a much more demanding radiation environment at the future high-luminosity LHC (HL-LHC) compared to LHC design values. The heart of the HEC read-out electronics is the pre-amplifier and summing (PAS) system which is realized in GaAs ASIC technology. The PAS devices are installed inside the LAr cryostat directly on the detector. They have been proven to operate reliably in LHC conditions up to luminosities of $1000 \mathrm{fb}^{-1}$, within safety margins. However, at the HL-LHC a total luminosity of $3000 \mathrm{fb}^{-1}$ is expected, which corresponds to radiation levels being increased by a factor 3-5. On top of that a safety factor needs to be accounted for to reflect our confidence in the simulations. The GaAs ASIC has therefore been exposed to neutron and proton radiation with integrated fluences in excess of $4 \cdot 10^{15} \mathrm{n} / \mathrm{cm}^{2}$ and 2.6 $10^{14}$ protons $/ \mathrm{cm}^{2}$, several factors above the levels corresponding to ten years of HL-LHC running. In-situ measurements of S-parameters allow the evaluation of frequency dependent performance parameters, like gain and input impedance. The non-linearity of the ASIC response has been measured both at warm and at cold, i.e. at LAr temperatures. This allows an improved estimation of the expected degradation of the HEC performance. The measured gain and nonlinearity of the ASIC response were finally applied to Monte-Carlo simulations in order to understand their effect on jet measurements in HL-LHC conditions.
\end{abstract}

Technology and Instrumentation in Particle Physics 2014

2-6 June, 2014

Amsterdam, the Netherlands 


\section{Introduction}

ATLAS [1] is a general-purpose detector at the Large Hadron Collider (LHC) designed for studying $p p$-collisions at centre-of-mass energies up to of $14 \mathrm{TeV}$ and an instantaneous luminosity of $10^{34} \mathrm{~cm}^{-2} \mathrm{~s}^{-1}$. A schematic view of the ATLAS liquid argon calorimeter system is presented in Fig. 1. The calorimetry played a major role in discovery of the Higgs boson [2]. The Higgs properties are planned to be further investigated at HL-LHC [3] which will run at five times higher luminosity aiming to provide statistics of $3000 \mathrm{fb}^{-1}$ by 2030 .

There are several options for the ATLAS end-cap upgrade [4], depending in particular on degradation of readout electronics (HEC preamplifier and summing boards, PSBs) mounted inside the cryostat at the outer surface of the HEC modules. The opening of the cryostat is a risky operation which one could avoid only if a satisfactory performance of the HEC electronics for HL-LHC operation is assured.

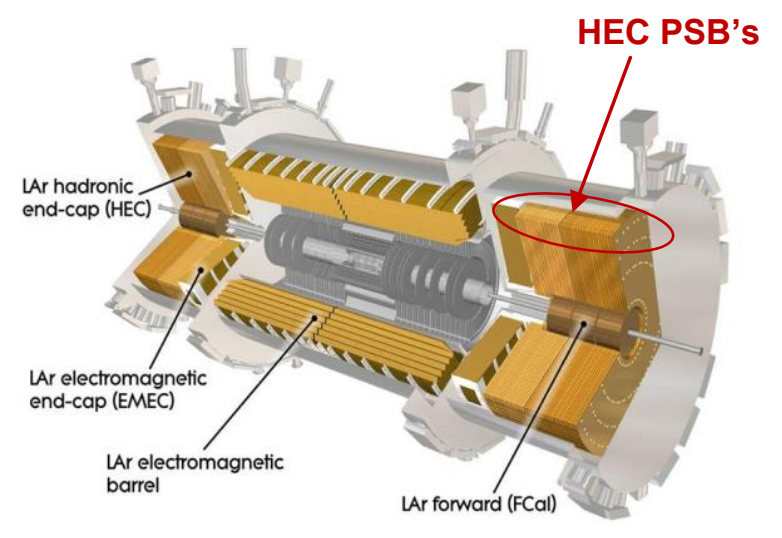

Figure 1: Schematic view of the ATLAS liquid argon calorimeter system. The marked area shows the radial location of the hadronic endcap readout electronics.

Fig. 2 represents the expected fluence in the ASICs of the HEC electronics. The HEC is segmented into four layers constructed in parallelplate geometry. It has copper absorbers and copper and kapton

electrodes in the liquid argon gaps. The color coded values were estimated for the integrated luminosity of $3000 \mathrm{fb}^{-1}$. A safety factor of 5 was introduced to account for simulation uncertainties

This study is aimed on making predictions of the HEC performance based on the irradiation tests results and radiation doses expected at the location of readout electronics. The results are crucial for final selection of the HEC upgrade option.

\section{Irradiation tests}

The HEC PSBs are equipped with GaAs ASICs. A series of neutron and proton irradiation tests have been undertaken on these ASICs at the Nuclear Physics Institute (NPI) in Řež, Czech Republic, and at the Paul Scherrer Institute (PSI) in Villigen, Switzerland [5]. Three different transistor technologies were tested: Si CMOS FET in SGB25V 250nm technology from IHP, SiGe Bipolar HBT (IHP SGB25V $250 \mathrm{~nm}$ and IBM 8WL-BiCMOS $130 \mathrm{~nm}$ ), and the GaAs FET currently used in the HEC, both the Triquint CFH800 250nm transistors themselves and integrated into the HEC BB96 Preamplifiers (PAs) and Systems [6]. The integrated fluences for $1 \mathrm{MeV}$ neutron equivalent $\left(4.1 \cdot 10^{15} \mathrm{n}_{\mathrm{eq}} / \mathrm{cm}^{2}\right)$ and proton radiation $\left(2.6 \cdot 10^{14}\right.$ protons $\left./ \mathrm{cm}^{2}\right)$ were 
several factors above the levels corresponding to ten years of HL-LHC running $\left(3000 \mathrm{fb}^{-1}\right)$. Insitu measurements of S-parameter using a Vector Network Analyzer allow the evaluation of frequency dependent performance parameters, like gain and input impedance, as a function of fluence. The irradiation and first analyses were taken in ambient conditions, while the Sparamter measurements were repeated at cold, i.e. at LAr temperatures. This allowed an improved estimation of the expected degradation of the HEC performance.

Fig. 3 (left) [7] demonstrates the gain degradation (forward transmission coefficient, $\mathbf{S}_{21}$ ) of the BB96 Systems. The red dashed line indicates the level of integrated dose expected at HLLHC assuming a safety factor of 5. For this dose, one may expect $20 \%$ losses of the calorimeter signal. Fig.3 (right) shows relative non-linearity of the readout electronics which is characterized by deviation of a power-law exponent from unity.
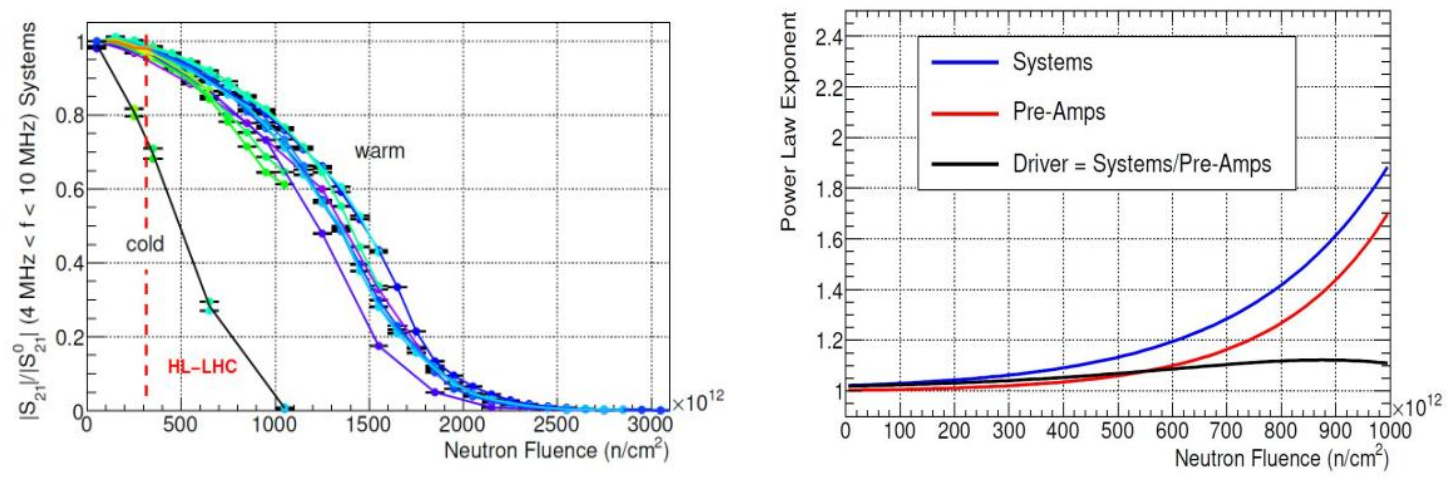

Figure 3: Forward transmission coefficient, $\left|S_{21}\right|$, of the BB96 Systems normalized to the value before irradiation (left), and linearity of the BB96 preamplifiers in terms of a power-law exponent (right).

Results of the irradiation tests were used as input parameters to estimate the HEC calorimeter performance for jet reconstruction. For the subset of simulated high- $\mathrm{p}_{\mathrm{T}}$ di-jet events the initial values of visible energy deposited in the liquid argon gaps of the HEC were substituted by the "degraded" ones and parameters of the reconstructed jets were compared for both cases. The preliminary results showed significant deterioration of the HEC performance if a safety factor value of 5 is assumed: e.g. for $E_{\text {jet }}>400 \mathrm{GeV}$ a degradation of the jet energy resolution by $4 \%$ and a degradation of linearity by $8 \%$ were observed.

New estimates of the neutron fluences expected in the HEC calorimeter were recently validated for a more realistic simulated detector geometry with a revised material budget and cavern background measurements allowing a reduction of the background simulation uncertainty to a factor of 2. Further studies are on-going in order to decide if the GaAs ASICs are still satisfactory for HL-LHC operation.

\begin{tabular}{|lcc|}
\hline Technology & Proton fluence & Neutron fluence \\
& $5.1 \cdot 10^{13}$ protons $/ \mathrm{cm}^{2}$ & $4.1 \cdot 10^{14} \mathrm{n}_{\text {eq }} / \mathrm{cm}^{2}$ \\
Si CMOS FET & $-3 \%$ & $0 \%$ \\
SiGe Bipolar & $-3 \%$ & $-1 \%$ \\
GaAs FET & $\mathrm{n} / \mathrm{a}$ & $-5 \%$ \\
GaAs BB96 (warm) & $-5 \%$ & $-8 \%$ \\
\hline
\end{tabular}

Table: Average gain degradation for GaAs technology currently used in ATLAS and alternative technologies after irradiation to the expected HL-LHC radiation levels. 
Tests of different transistor technologies revealed suitable candidates for the future electronics upgrade, if necessary. Results for the gain degradation as measured in the irradiation tests at NPI (neutrons) and PSI (protons) are presented in the Table.

\section{Conclusions}

A comprehensive program of irradiation tests with protons and neutrons has been performed on the readout electronics currently installed in the endcap cryostat of the ATLAS detector. Testing of the different alternative technologies (Si CMOS FET and SiGe Bipolar) demonstrated promising results for those upgrade options where "cold" electronics replacement is involved.

The study of the HEC performance is on-going by implementing recently validated values of the expected doses and corresponding degradation effects, which were observed in the irradiation tests, in di-jet events simulation. Very preliminary results for the a safety factor of 2 show a modest (acceptable) deterioration of the jet energy resolution and linearity. Hopefully, the risky option of the HEC upgrade which includes the cryostat opennig will not be claimed.

\section{References}

[1] ATLAS Collaboration, "The ATLAS experiment at the CERN Large Hadron Collider", JINST, 3, S08003, 2008.

[2] ATLAS Collaboration, "First observation of a new particle in the search for the Standard Model Higgs boson at the LHC", Phys. Lett. B716 (2012) 1-29

[3] L. Rossi and O. Bruning, "High Luminosity Large Hadron Collider: A description for the European Strategy Preparatory Group”, CERN-ATS-2012-236; http://cds.cern.ch/record/1471000

[4] ATLAS Collaboration, "Letter of Intent for the Phase-II Upgrade of the ATLAS Experiment", CERN, Geneva, CERN-LHCC-2012-022, Dec. 2012; https://cds.cern.ch/record/1502664

[5] M. Nagel on behalf of the ATLAS Liquid Argon Calorimeter Group and the HECPAS Collaboration, "Irradiation Tests and Expected Performance of Readout Electronics of the ATLAS Hadronic Endcap Calorimeter for the HL-LHC", arXiv:1309.0672 [physics.ins-det]

[6] J. Ban, H. Brettel, W. Cwienk, J. Fent, L. Kurchaninov, et al., "Cold electronics for the liquid argon hadronic endcap calorimeter of ATLAS”, NIM A556 (2006) 158.

[7] S. Menke. Proton and Neutron Irradiation Tests of Readout Electronics of the ATLAS Hadronic Endcap Calorimeter. arXiv:1211.4060,2011 